\title{
NUMERICAL DATA FOR WIND TURBINE MICROSITING INSPIRED BY HUMAN DYNASTIES BY USE OF THE DYNASTIC OPTIMIZATION ALGORITHM (DOA)
}

\author{
Shafiq-ur-Rehman Massan \\ QEC and Coordination \\ Mohammad Ali Jinnah University, Karachi and \\ Shaheed Zulfikar Ali Bhutto Institute of Science and Technology, Karachi, (Pakistan). \\ E-mail: srmassan@hotmail.com ORCID: https://orcid.org/0000-0001-6548-6513
}

\begin{abstract}
Asim Imdad Wagan
Department of Engineering

Mohammad Ali Jinnah University, Karachi, (Pakistan).

E-mail: aiwagan@gmail.com ORCID: https:// orcid.org/0000-0001-9765-5385
\end{abstract}

Muhammad Mujtaba Shaikh

Department of Basic Sciences and Related Studies

Mehran University of Engineering and Technology, Jamshoro, (Pakistan). E-mail: mujtaba.shaikh@faculty.muet.edu.pk ORCID: https://orcid.org/0000-0002-1471-822X

Citación sugerida:

Massan, S.-U.-R., Wagan, A. I., y Shaikh, M. M. (2020). Numerical data for wind turbine micrositing inspired by human dynasties by use of the Dynastic Optimization Algorithm (DOA). 3C Tecnología. Glosas de innovación aplicadas a la pyme, 9(2), 71-85. http://doi.org/10.17993/3ctecno/2020.v9n2e34.71-85 


\section{ABSTRACT}

This work presents the newly formulated Dynastic Optimization Algorithm, DOA as applied to the wind turbine micrositing problem. The data is acquired by the use of the standard MATLAB software at a wind speed of $12 \mathrm{~m} / \mathrm{s}$. The values of the efficiency of the algorithm, cost per installation of per unit turbine, and total dissipated power at each number of turbines installed are discussed.

This algorithm is applied to two test functions and the results are described therein. It has been welldemonstrated that the proposed DOA exhibits superior performance over GA and DEA for test functions by hitting the minima very often and with higher precision. On the other hand DOA performance on WTM problem is also encouraging.

\section{KEYWORDS}

Dynastic Optimization Algorithm (DOA), Metaheuristic Algorithms, Genetic Algorithm (GA), Differential Evolution Algorithm (DEA), Wind Turbine Micrositing (WTM). 


\section{INTRODUCTION}

This work is inspired by the works of Grady, Hussaini, and Abdullah (2005), Mosetti, Poloni, and Diviacco (1994), Emami and Nougreh (2010) and Marmidis, Lazarou, and Pyrgioti (2008).

The data in this article has been compared with similar results of Mittal (2010), Rajper and Amin (2012) and Massan, Wagan, \& Shaikh (2020). The nature-inspired algorithms use the best combination and evolution strategy in a given situation. In this work, a new metaheuristic algorithm is developed by using social behavior in human dynasties. The motivation, conceptual framework, mathematical model, pseudocode and working of the algorithm are described in this paper and the adjoining papers. The proposed dynastic optimization algorithm (DOA, which is the base paper supporting this data. Comparison was also made to similar studies (Massan, Wagan, \& Shaikh, 2017; Massan et al., 2015; Massan et al., 2017a, 2017b).

The effect of wind speed on the resultant power output on an ascending number of turbines arranged by the metaheuristic method of the Dynastic Optimization Algorithm in a wind farm is evaluated. A new metaheuristic algorithm for wind form micrositing known as "Dynastic optimization algorithm" (DOA) was discussed in Massan, Wagan, and Shaikh (2020). The nature-inspired algorithms use the best combination and evolution strategy in a given situation. In this work, a new metaheuristic algorithm is developed by using social behavior in human dynasties. The motivation, conceptual framework, mathematical model, pseudocode and working of the algorithm are described in this paper and the adjoining papers. The proposed dynastic optimization algorithm (DOA, and the important data about the power produced, cost per unit turbine installation and efficiency of DOA are shared in this data article. The complete methodology of DOA can be found in Massan, Shaikh, \& Wagan (2020). The data is summarized in Table 1 and Figures 1-3. 


\section{OBJECTIVES}

The work describes the data obtained for a novel algorithm that has been presented in Massan, Wagan and Shaikh (2020). The nature-inspired algorithms use the best combination and evolution strategy in a given situation. In this work, a new metaheuristic algorithm is developed by using social behavior in human dynasties. The motivation, conceptual framework, mathematical model, pseudocode and working of the algorithm are described in this paper and the adjoining papers. The proposed dynastic optimization algorithm (DOA and puts it forward for wider scientific use. It is evident that it shall prove to be useful while comparing with other algorithms as applied to this problem and other similar problems.

\section{EXPERIMENTAL DESIGN, MATERIALS AND METHODS}

The method is described in Massan, Shaikh, and Wagan (2020) and the following parameters have been used to carry out the simulations.

Using the below defined parameters from Massan, Wagan and Shaikh (2020). The nature-inspired algorithms use the best combination and evolution strategy in a given situation. In this work, a new metaheuristic algorithm is developed by using social behavior in human dynasties. The motivation, conceptual framework, mathematical model, pseudocode and working of the algorithm are described in this paper and the adjoining papers. The proposed dynastic optimization algorithm (DOA, and the methodology from Massan, Shaikh, and Wagan (2020), the numerical data concerning the power produced in (Kwh), cost per unit turbine installation (dimensionless), and the efficiency (per unit) of DOA application is shared in table 1 for the installation of 100 turbines. 


\section{DATA ANALYSIS}

The data was acquired by use of a Corei7 laptop ( $7^{\text {th }}$ generation) and the runtime was less than 8 hours for Matlab 2017, student version. The data format is raw and analyzed. The parameter values are as per the given Table 1.

Table 1. Parameters used for DOA implementation.

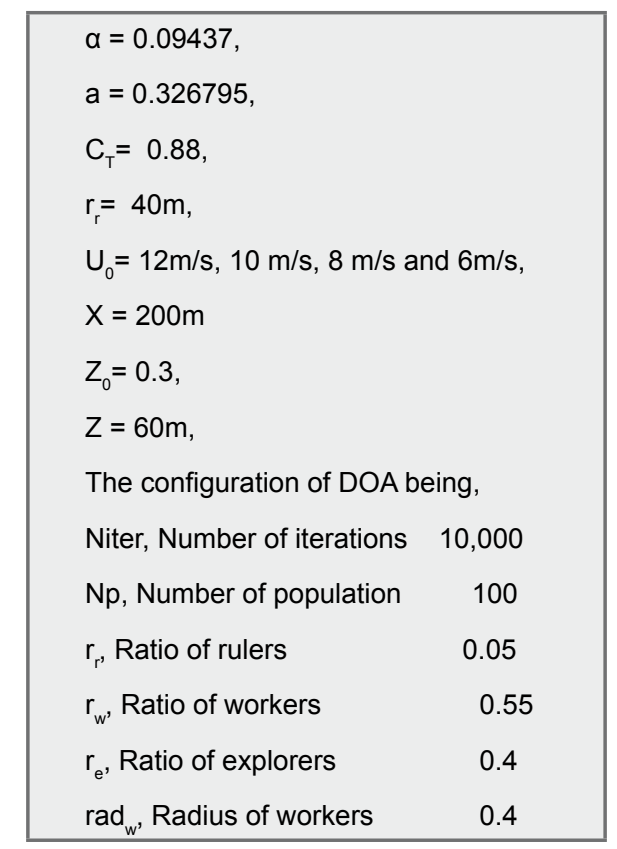

The value of the data is that it depicts the actual implementation of a new algorithm for the computation of the WTM problem. It shall save the computation time for other researchers and shall be a viable source of comparison of other similar research and application of other algorithms.

This algorithm is competing with other algorithms such as the GA and DEA which are in wide use. The results are obtained by the use of the same code as used by Mittal (2010) and the data analysis methods utilized in Sultan, Shaikh, and Chowdhry (2020). 
The submission of results of a new algorithm in this domain opens new avenues for research and provides a base for comparison with standard benchmark algorithms. These results shall provide the basis of scientific testing of the DOA algorithm.

Table 2. Dynastic Optimization Algorithm, Results of power, cost, and efficiency per unit turbine.

\begin{tabular}{|c|c|c|c|}
\hline \# of Turbines & Power by DOA & Cost by DOA & Efficiency by DOA \\
\hline 1 & 518.4 & 0.001927894 & 1 \\
\hline 2 & $1,036.80$ & 0.001924553 & 1 \\
\hline 3 & $1,555.20$ & 0.001919021 & 1 \\
\hline 4 & $2,073.60$ & 0.001911358 & 1 \\
\hline 5 & $2,592.00$ & 0.001901641 & 1 \\
\hline 6 & $3,110.40$ & 0.00188997 & 1 \\
\hline 7 & $3,628.80$ & 0.001876462 & 1 \\
\hline 8 & $4,147.20$ & 0.00186125 & 1 \\
\hline 9 & $4,665.60$ & 0.001844484 & 1 \\
\hline 10 & $5,184.00$ & 0.001826323 & 1 \\
\hline 11 & $5,702.40$ & 0.001806936 & 1 \\
\hline 12 & $6,220.80$ & 0.0017865 & 1 \\
\hline 13 & $6,739.20$ & 0.001765195 & 1 \\
\hline 14 & $7,257.60$ & 0.001743204 & 1 \\
\hline 15 & $7,776.00$ & 0.001720706 & 1 \\
\hline 16 & $8,294.40$ & 0.00169788 & 1 \\
\hline 17 & $8,812.80$ & 0.001674896 & 1 \\
\hline 18 & $9,328.22$ & 0.001652447 & 0.999680735 \\
\hline 19 & $9,845.28$ & 0.00162982 & 0.999561186 \\
\hline 20 & $10,359.23$ & 0.001607955 & 0.999153779 \\
\hline 21 & $10,880.17$ & 0.001585428 & 0.999427594 \\
\hline 22 & $11,394.89$ & 0.001564361 & 0.999130908 \\
\hline 23 & $11,909.58$ & 0.001543903 & 0.998857632 \\
\hline 24 & $12,429.13$ & 0.001523553 & 0.998998052 \\
\hline 25 & $12,805.65$ & 0.00152085 & 0.988090649 \\
\hline 26 & $13,453.78$ & 0.00148705 & 0.998173546 \\
\hline
\end{tabular}




\begin{tabular}{|c|c|c|c|}
\hline \# of Turbines & Power by DOA & Cost by DOA & Efficiency by DOA \\
\hline 27 & $13,969.90$ & 0.001469687 & 0.998078289 \\
\hline 28 & $14,485.15$ & 0.001453366 & 0.997929592 \\
\hline 29 & $14,996.40$ & 0.001438399 & 0.997525317 \\
\hline 30 & $15,514.40$ & 0.00142376 & 0.997582357 \\
\hline 31 & $16,027.31$ & 0.001410575 & 0.997318922 \\
\hline 32 & $16,559.21$ & 0.001396744 & 0.99821631 \\
\hline 33 & $17,053.30$ & 0.001387048 & 0.996849034 \\
\hline 34 & $17,562.23$ & 0.00137699 & 0.996404884 \\
\hline 35 & $18,066.46$ & 0.001368154 & 0.995726221 \\
\hline 36 & $18,573.76$ & 0.001359898 & 0.995250507 \\
\hline 37 & $19,097.40$ & 0.001351271 & 0.995652038 \\
\hline 38 & $19,596.40$ & 0.00134515 & 0.994781272 \\
\hline 39 & $20,131.35$ & 0.0013373 & 0.995733981 \\
\hline 40 & $20,640.35$ & 0.001331884 & 0.995387307 \\
\hline 41 & $21,144.41$ & 0.001327386 & 0.994825064 \\
\hline 42 & $21,664.12$ & 0.001322477 & 0.995008576 \\
\hline 43 & $22,179.64$ & 0.001318368 & 0.994995257 \\
\hline 44 & $22,666.38$ & 0.001316417 & 0.993721187 \\
\hline 45 & $23,181.67$ & 0.001313211 & 0.993727436 \\
\hline 46 & $23,667.78$ & 0.001312025 & 0.992509534 \\
\hline 47 & $24,178.36$ & 0.001309801 & 0.992348055 \\
\hline 48 & $24,685.19$ & 0.001308089 & 0.992042415 \\
\hline 49 & $25,194.62$ & 0.001306513 & 0.991851631 \\
\hline 50 & $25,697.39$ & 0.00130552 & 0.991411503 \\
\hline 51 & $26,203.16$ & 0.001304578 & 0.991102259 \\
\hline 52 & $26,719.00$ & 0.001303325 & 0.991178533 \\
\hline 53 & $27,210.82$ & 0.001303398 & 0.99037745 \\
\hline 54 & $27,748.88$ & 0.001301409 & 0.991258057 \\
\hline 55 & $28,193.69$ & 0.001303894 & 0.988835927 \\
\hline 56 & $28,753.09$ & 0.001301182 & 0.990447563 \\
\hline 57 & $29,130.48$ & 0.001306762 & 0.985843065 \\
\hline
\end{tabular}




\begin{tabular}{|c|c|c|c|}
\hline \# of Turbines & Power by DOA & Cost by DOA & Efficiency by DOA \\
\hline 58 & $29,687.59$ & 0.001304321 & 0.987374528 \\
\hline 59 & $30,242.16$ & 0.001302135 & 0.988771037 \\
\hline 60 & $30,741.34$ & 0.001302418 & 0.988340485 \\
\hline 61 & $31,278.57$ & 0.001301147 & 0.989126935 \\
\hline 62 & $31,640.64$ & 0.00130715 & 0.984438589 \\
\hline 63 & $32,200.11$ & 0.001304997 & 0.985943057 \\
\hline 64 & $32,642.62$ & 0.00130761 & 0.98387515 \\
\hline 65 & $33,123.50$ & 0.001308655 & 0.983009914 \\
\hline 66 & $33,632.55$ & 0.001308591 & 0.982994074 \\
\hline 67 & $34,173.13$ & 0.001307335 & 0.983886443 \\
\hline 68 & $34,195.85$ & 0.001325909 & 0.970061967 \\
\hline 69 & $35,102.20$ & 0.001310625 & 0.981341563 \\
\hline 70 & $35,651.06$ & 0.001309114 & 0.982447598 \\
\hline 71 & $36,224.84$ & 0.001306755 & 0.984199447 \\
\hline 72 & $36,637.20$ & 0.001310223 & 0.981577858 \\
\hline 73 & $37,072.03$ & 0.001312822 & 0.979621874 \\
\hline 74 & $37,613.83$ & 0.001311622 & 0.980507256 \\
\hline 75 & $38,080.59$ & 0.001313042 & 0.979439083 \\
\hline 76 & $38,671.75$ & 0.001310201 & 0.981556231 \\
\hline 77 & $39,084.93$ & 0.001313401 & 0.979159875 \\
\hline 78 & $39,661.54$ & 0.00131111 & 0.98086661 \\
\hline 79 & $39,516.82$ & 0.001332779 & 0.964916909 \\
\hline 80 & $40,750.09$ & 0.0013088 & 0.982592726 \\
\hline 81 & $41,107.04$ & 0.001313651 & 0.97896282 \\
\hline 82 & $41,465.55$ & 0.001318369 & 0.975458058 \\
\hline 83 & $42,185.95$ & 0.001311657 & 0.980448512 \\
\hline 84 & $42,506.64$ & 0.001317444 & 0.976140856 \\
\hline 85 & $43,123.26$ & 0.001314065 & 0.978650658 \\
\hline 86 & $43,316.21$ & 0.001323602 & 0.971598857 \\
\hline 87 & $43,863.89$ & 0.001322274 & 0.972574453 \\
\hline 88 & $44,328.75$ & 0.001323446 & 0.971712521 \\
\hline
\end{tabular}




\begin{tabular}{|c|c|c|c|}
\hline \# of Turbines & Power by DOA & Cost by DOA & Efficiency by DOA \\
\hline 89 & $44,977.38$ & 0.001319182 & 0.974852994 \\
\hline 90 & $45,593.26$ & 0.001315985 & 0.977221701 \\
\hline 91 & $45,646.31$ & 0.00132906 & 0.967607595 \\
\hline 92 & $46,039.29$ & 0.001332196 & 0.965330019 \\
\hline 93 & $46,885.14$ & 0.001322381 & 0.972494843 \\
\hline 95 & $47,024.66$ & 0.001332634 & 0.965012274 \\
\hline 96 & $47,563.55$ & 0.001331552 & 0.965796586 \\
\hline 97 & $48,202.02$ & 0.001327745 & 0.968565566 \\
\hline 99 & $48,753.12$ & 0.001326411 & 0.969539892 \\
\hline 100 & $48,430.18$ & 0.001349021 & 0.953290011 \\
\hline
\end{tabular}

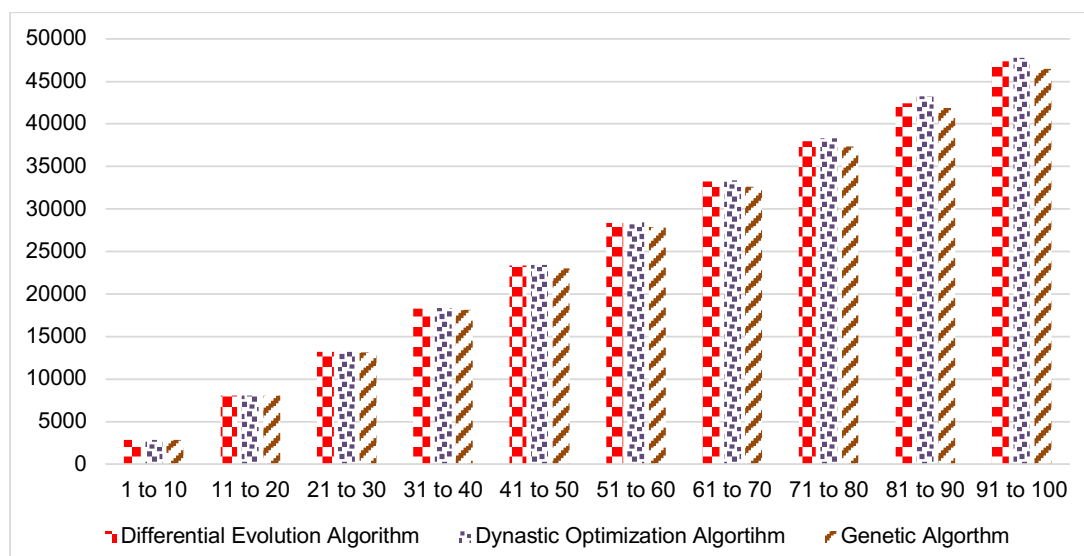

Figure 1. Comparison of mean power (kWh) produced by DEA (Massan et al., 2017a, 2017b), DOA (Massan, Wagan, \& Shaikh, 2020). The nature-inspired algorithms use the best combination and evolution strategy in a given situation. In this work, a new metaheuristic algorithm is developed by using social behavior in human dynasties. The motivation, conceptual framework, mathematical model, pseudocode and working of the algorithm are described in this paper and the adjoining papers. The proposed dynastic optimization algorithm (DOA and GA (Rajper \& Amin, 2012) versus number of turbines. 




Figure 2. Comparison of mean cost per unit turbine (dimensionless) by DEA (Massan et al., 2017a, 2017b), DOA (Massan, Wagan, \& Shaikh, 2020). The nature-inspired algorithms use the best combination and evolution strategy in a given situation. In this work, a new metaheuristic algorithm is developed by using social behavior in human dynasties. The motivation, conceptual framework, mathematical model, pseudocode and working of the algorithm are described in this paper and the adjoining papers. The proposed dynastic optimization algorithm (DOA and GA (Rajper \& Amin, 2012) versus number of turbines.

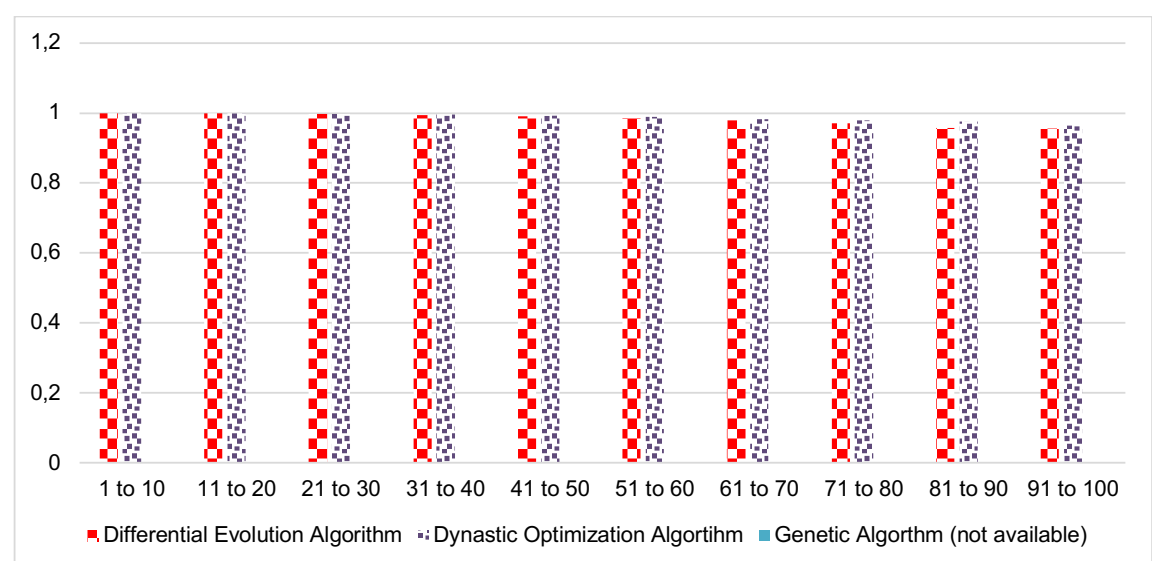

Figure 3. Comparison of efficiencies (per unit) by DEA (Massan et al., 2017a, 2017b), DOA (Massan, Wagan, \& Shaikh, 2020). The nature-inspired algorithms use the best combination and evolution strategy in a given situation. In this work, a new metaheuristic algorithm is developed by using social behavior in human dynasties. The motivation, conceptual framework, mathematical model, pseudocode and working of the algorithm are described in this paper and the adjoining papers. The proposed dynastic optimization algorithm (DOA and GA (Rajper \& Amin, 2012) versus number of turbines. 


\section{TEST FUNCTIONS}

This algorithm was applied to the following two test functions and the comparative graphs are obtained herewith,

The DOA, DEA and GA were applied to the following test functions,

Booths's $\left(\mathrm{f}_{1}\right)$ and

$$
f_{1}\left(x_{1}, x_{2}\right)=\left(x_{1}+2 x_{2}-7\right)^{2}+\left(2 x_{1}+x_{2}-5\right)^{2}
$$

the Bohachevsky's (f2) functions

$$
f_{2}\left(x_{1}, x_{2}\right)=-x_{1}^{2}+2 x_{2}^{2}-0.3 \cos \left(3 \pi x_{1}\right)-0.4 \cos \left(4 \pi x_{2}\right)+0.7
$$

The following figures were obtained,
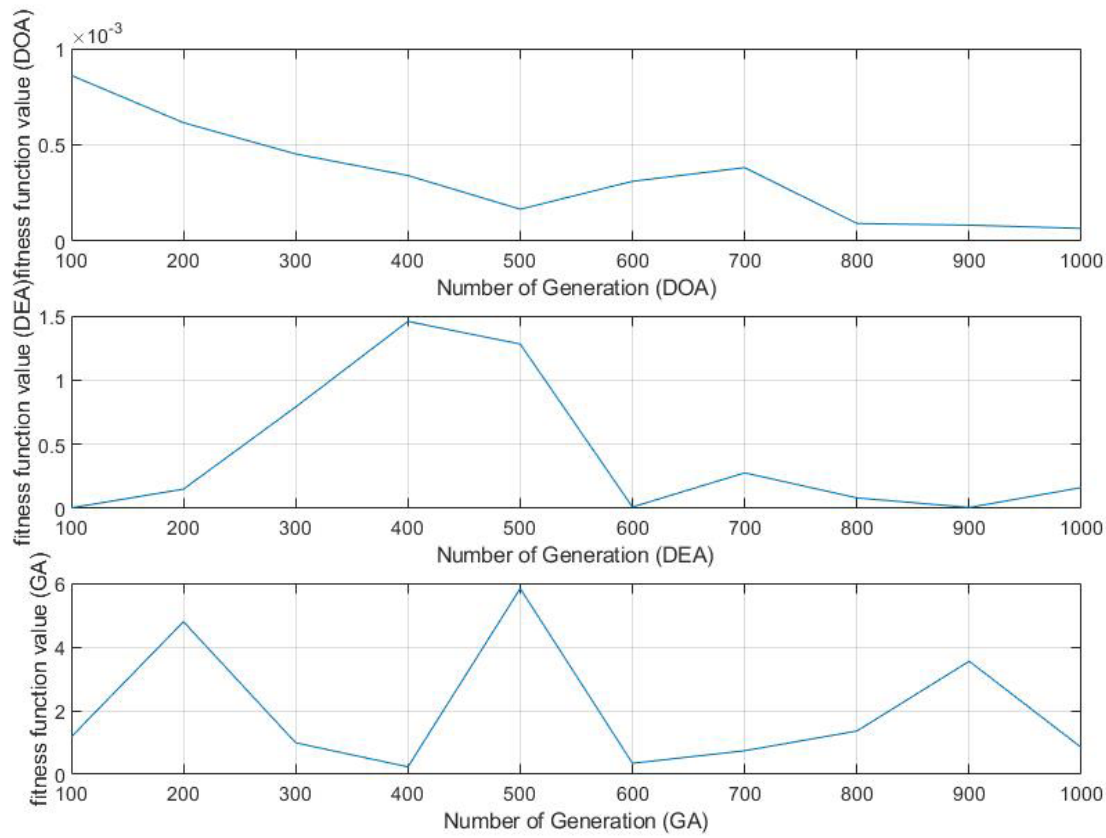

Figure 4. Comparison of minima attained versus number of generations by all methods for Booth's function. 

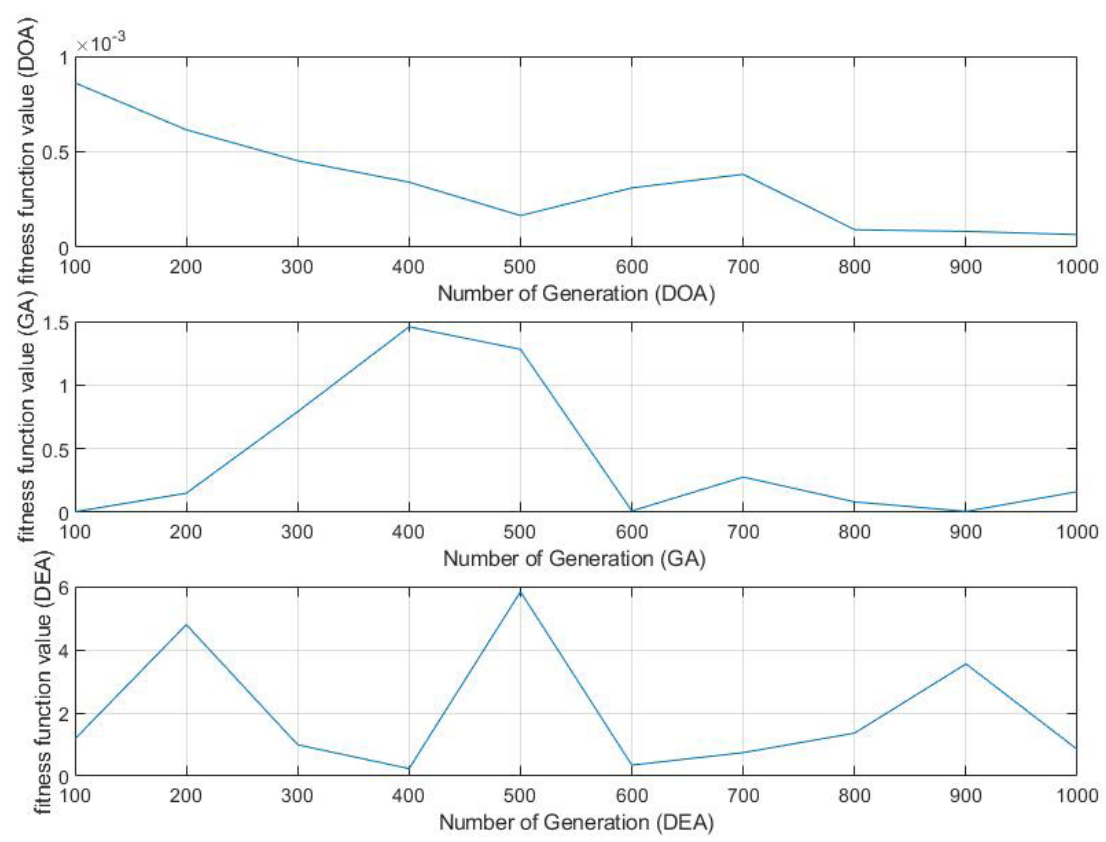

Figure 5. Comparison of minima attained versus number of generations by all methods for Bohachevsky's function.

The minimum value of the Booth's function is 0 at $(1,3)$ and the minimum value of the Bohachevsky's function is 0 at $(0,0)$.

The DOA approaches the minima of $\mathrm{fl}$ and $\mathrm{f} 2$ more frequently and with comparatively much higher precision than GA and DEA as demonstrated through Figures 4 and 5 for a several values of generations.

\section{RECOMMENDATIONS}

In view of the encouraging results of the DOA algorithm it is now possible to depict that it is a viable algorithm that may be used in different fields of technology. The values of the test functions also depict encouraging results for this algorithm. 


\section{CONCLUSION}

The potential power saving, cost saving and efficiency benefits of proposed DOA (Massan, Wagan, and Shaikh, 2020). The nature-inspired algorithms use the best combination and evolution strategy in a given situation. In this work, a new metaheuristic algorithm is developed by using social behavior in human dynasties. The motivation, conceptual framework, mathematical model, pseudocode and working of the algorithm are described in this paper and the adjoining papers. The proposed dynastic optimization algorithm (DOA are shown in Figures 1-3, respectively against Differential Evolution Algorithm (Massan et al., 2017a, 2017b) and genetic algorithm data (Rajper \& Amin, 2012). The encouraging performance of DOA over GA and DEA is evident from the exhaustive comparison in Massan, Wagan, and Shaikh (2020). The nature-inspired algorithms use the best combination and evolution strategy in a given situation. In this work, a new metaheuristic algorithm is developed by using social behavior in human dynasties. The motivation, conceptual framework, mathematical model, pseudocode and working of the algorithm are described in this paper and the adjoining papers. The proposed dynastic optimization algorithm (DOA and the data shared in this article.

\section{ACKNOWLEDGEMENT}

The authors wish to thank Hazrat Manzoor Hussain (RA) for his guidance and support.

\section{REFERENCES}

Emami, A., \& Nougreh, P. (2010). New approach on optimization in placement of wind turbines within wind farm by genetic algorithms. Renewable Energy, (35), 169-178. https://doi.org/10.1016/j. renene.2009.11.026

Grady, S. A., Hussaini, M. Y., \& Abdullah, M. M. (2005). Placement of wind Turbines using genetic algorithms. Renewable Energy, I(30), 259-270. https://doi.org/10.1016/j.renene.2004.05.007 
Marmidis, G., Lazarou, S., \& Pyrgioti, E. (2008). Optimal placement of wind turbines in a wind park using Monte Carlo simulation. Renewable Energy, (33), 1455-1460. https:/ / doi.org/10.1016/j. renene.2007.09.004

Massan, S.-R., Shaikh, M. M., \& Wagan, A. I. (2020). The method of propagation of the Dynastic Optimization Algorithm within the wind turbine optimization problem. MethodX (Submitted).

Massan, S.-R., Wagan, A. I., \& Shaikh, M. M. (2017). A New Hybrid Metaheuristic Algorithm for Wind Farm Micrositing. Mehran University Research fournal Of Engineering \& Technology, 36(3), 635-648. http://publications.muet.edu.pk/article_detail_abstract.php?p_id=1574

Massan, S.-R., Wagan, A. I., \& Shaikh, M. M. (2020). A new metaheuristic optimization algorithm inspired by human dynasties with an application to the wind turbine micrositing problem. Applied Soft Computing, 90, 106176. https://doi.org/10.1016/J.ASOG.2020.106176

Massan, S.-R., Wagan, A. I., Shaikh, M. M., \& Abro, R. (2015). Wind turbine micrositing by using the firefly algorithm. Applied Soft Computing, 27, 450-456. https://doi.org/10.1016/j. asoc.2014.09.048

Massan, S.-R., Wagan, A. I., Shaikh, M. M., \& Shah, M. S. (2017a). Application of Differential Evolution Algorithm for Wind Turbine Micrositing. Mehran University Research fournal Of Engineering E Technology, 36(2), 133-146. http://publications.muet.edu.pk/

Massan, S.-U.-R., Wagan, A. I., Shaikh, M. M., \& Shah, M. S. (2017b). Numerical data concerning wind farm layout optimization using differential evolution algorithm at different wind speeds. Data in Brief, 15, 244-248. https://doi.org/10.1016/j.dib.2017.09.040

Mittal, A. (2010). Optimization of the layout of large wind farms using genetic algorithm. (MS Thesis). Department of Aerospace and Mechanical Engineering, Case Western Reserve University, USA. Case Western Reserve University. https://doi.org/10.1002/fld 
Mosetti, G., Poloni, G., \& Diviacco, B. (1994). Optimization of wind farms positioning in large wind farms by means of a genetic algorithm. Wind Engineering and Industrial Aerodynamics, (51), 105-1 16. https://doi.org/10.1016/0167-6105(94)90080-9

Rajper, S., \& Amin, I. J. (2012). Optimization of wind turbine micrositing: A comparative study. Renewable and Sustainable Energy Reviews, 16, 5485-5492.https://doi.org/10.1016/j.rser.2012.06.014

Sultan, M., Shaikh, M. M., \& Ghowdhry, N. P. (2020). Comparative Analysis of Knee Joint Replacement and Stem Cells Therapy Treatment for Knee Osteoarthritis Using Statistical Techniques. Research in Medical and Engineering Sciences, 10(4), 887-897. https: / /www.researchgate. net/publication/339415539_Comparative_Analysis_of_Knee_Joint_Replacement_and_Stem_ Cells_Therapy_Treatment_for_Knee_Osteoarthritis_Using_Statistical_Techniques 